\title{
Uma avaliação da demanda creditícia para automóveis no Brasil no período de 2000 a 2012 *
}

\author{
Mário Jorge Mendonça ** \\ Tito Belchior S. Moreira ${ }^{* * *}$ \\ Adolfo Sachsida ${ }^{* * * *}$
}

\section{Resumo}

Este estudo tem como objetivo estimar uma função demanda por crédito para veículos no Brasil no período 2000:06 a 2012:12. Nossos resultados mostraram que essa função pode ser enquadrada dentro de um arcabouço tradicional no qual a taxa e prazo de financiamento, o preço do veículo assim como o estado da economia se apresentam como as variáveis explicativas do modelo. Mostramos ainda com base no modelo Markov Switching (MS) com variável endógena (Kim, 2004) que tal função de demanda esteve sujeita a três regimes distintos ao longo do período amostral. Dois desses estados estão associados com regimes de expansão e retração inerentes as fases de uma economia de mercado no qual o estado da economia é determinado pelos seus fundamentos. Um ciclo de forte expansão acontece entre 2004 e 2007 enquanto um ciclo de retração vigora entre o final de 2007 e início de 2008 . O terceiro regime, também de expansão, acontece num único ciclo entre 2008 e 2009 sendo no nosso entendimento gerado de modo artificial em decorrência do emprego de medidas anticíclicas fomentadas pelo governo. Entre elas, pode-se destacar a redução da base de recolhimento do compulsório e da alíquota do IPI. Essas providências promoveram a imediata expansão do crédito e levaram a um processo de formação de bolha no mercado de veículos, que culminou com a necessidade de introdução das chamadas medidas macroprudenciais, a partir de dezembro de 2010, quando já havia em curso uma tendência de contínuo aumento da inadimplência no crédito.

Palavras-chave: Crédito para veículos; GMM; Modelo Markov Switching; Bolha de crédito; Inadimplência.

* Artigo recebido em 22 de outubro de 2014 e aprovado em 30 de março de 2017.

** Técnico de Planejamento e Pesquisa na Diretoria de Estudos Regionais, Ambientais e Urbanos (Dirur) do Instituto de Pesquisa Econômica Aplicada (Ipea), Brasília, DF, Brasil. E-mail: mario.mendonca@ipea.gov.br.

${ }^{* * * *}$ Professor e Pesquisador no Departamento de Economia da Universidade Católica de Brasília (UCB), Brasília, DF, Brasil. E-mail: tito@pos.ucb.br.

${ }^{* * * *}$ Técnico de Planejamento e Pesquisa na Dirur do Ipea, Brasília, DF, Brasil. E-mail: sachsida@hotmail.com. 


\section{Abstract \\ An evaluation of the automotive credit demand in from 2000 to 2012}

The aim of this study is to estimate the demand function for credit for vehicles in Brazil during the period between of July 2000 to December 2012. Our results showed that this function can be framed within a traditional framework in which the rate and the term of financing, the price of the vehicle as well as the state of the economy are the explanatory variables of the model. The Markov Switching (MS) model with an endogenous variable (Kim, 2004) showed that such a demand function was subject to three different regimes throughout the sample period. Two of these states are associated with expansion and retraction regimes in which the state of the economy is determined by its fundamentals. A cycle of strong expansion takes place between 2004 and 2007 while a cycle of retraction is in force between the end of 2007 and the beginning of 2008. The third regime, also of expansion, takes place in a single cycle between 2008 and 2009 and was artificially generated as a result of the use of countercyclical measures fomented by the government. Among them, we can highlight the reduction in the compulsory and the reserve requirement imposed by the Central Bank and the tax rate on the production of vehicles. These measures promoted the immediate expansion of credit and led to a process of bubble formation in the vehicle market. This culminated in the need to introduce the so-called macroprudential measures in December 2010, when there was already a continuous and growing trend in the credit default.

Keywords: Credit for vehicles; GMM; Markov Switching model; Credit bubble; Credit default.

JEL E32, R38.

\section{Introdução}

Diversos acontecimentos ocorridos a partir de 2000 contribuíram significativamente para o avanço do crédito no Brasil. Em meados desse ano, o saldo do crédito do sistema financeiro brasileiro correspondia a $28,3 \%$ do produto interno bruto (PIB). Em março de 2012, essa participação atingiu $53,48 \%$. Tais acontecimentos foram decorrentes de dois fatores principais. Primeiro, a introdução de reformas institucionais. A lei 10.913, que disciplinou a aplicação da alienação fiduciária ${ }^{1}$, aplicada originariamente ao mercado de capitais, foi estendida a outros mercados como de automóveis e imóveis. De acordo com Assunção, Benmelech e

(1) De acordo com esta lei, o banco, após a concessão de um empréstimo, detém a propriedade do bem e usa isto como garantia, cabendo ao mutuário o direito de uso. Quando o mutuário liquidar dívida, o banco transfere a propriedade do bem em definitivo para o mutuário. A lei foi aprovada em 2 de agosto de 2004. 
Silva (2012), essa reforma ampliou o crédito no setor de veículos, diminuindo o spread e aumentando o prazo de financiamento. Outra reforma introduzida foi a regulamentação do crédito consignado, que passou a vigorar a partir dezembro de 2003. Esta modalidade de crédito permite que o desconto da prestação seja feito diretamente na folha de pagamento ou benefício previdenciário, do cidadão ${ }^{2}$. Por fim, temos ainda a criação do Sistema de Informação de Crédito do Banco Central $^{3}$ (SCR), em maio de 2002, que deu mais segurança ao sistema financeiro. Por outro lado, os efeitos da estabilização da economia se fizeram sentir. Isso se refletiu no crescimento da economia, diminuição do desemprego e pelo aumento real da renda.

Entre os setores que mais se beneficiaram dessas mudanças se encontra o setor automobilístico. A ampliação do instituto da alienação fiduciária fez crescer o acesso ao crédito para segmentos de maior risco como, por exemplo, mutuários de baixa renda, que puderam ter acesso à compra de carros mais novos e de modelos com preços mais elevados. As reformas institucionais no crédito assim como o bom estado da economia propiciaram o surgimento de ciclo de prosperidade que se verificou a partir de 2004. De acordo com Araújo (2012), o ciclo de expansão de crédito, a partir de 2003, foi marcado por dois fatos distintos. Até a eclosão da crise financeira internacional de 2008-2009, a expansão do crédito foi liderada pelos bancos privados, que expandiram as operações de crédito a uma taxa superior à dos bancos públicos. Contudo, por conta dos efeitos da crise internacional sobre a economia brasileira sentidos mais intensamente a partir do final de 2008, observou-se a retomada do crescimento do crédito liderado pelo setor público com a elevação de $15,2 \%$ no saldo das operações de crédito do Sistema Financeiro Nacional (SFN) ${ }^{4}$.

(2) O empréstimo pessoal consignado em folha de pagamento, que não era representativo em 1999, alcançou, em março de 2011, R $\$ 143$ bilhões de saldo, ou o equivalente a 67,5\% de participação no crédito pessoal (que representava $18,8 \%$ do crédito livre).

(3) O SCR é um sistema de registro e consulta de informações sobre operações de crédito, avais e fianças e limites de crédito concedidos por instituições financeiras.

(4) Em 2009 houve uma expansão acentuada $(29,1 \%)$ do crédito direcionado e aumento da participação dos bancos públicos no total de crédito do SFN, que passou de 36,3\%, em 2008, para 41,5\%, em 2009. 
O setor automobilístico foi um dos grandes beneficiários das medidas anticíclicas adotadas pelo governo federal no intuito de mitigar os efeitos da crise. Um ponto que merece destaque é que o controle ou manipulação do crédito foi usado recorrentemente como instrumento de política econômica, sobretudo a partir de 2008, para mitigar o ciclo econômico. Entre essas medidas, encontram-se aquelas que tinham como objetivo aumentar a oferta de crédito para financiamento de veículos na medida em que foram facilitadas as condições para liberação de crédito como, por exemplo, a extensão do prazo de financiamento acima de 24 meses. Além disso, o governo procurou incentivar o mercado automobilístico diminuindo a alíquota do IPI.

Constatou-se que os incentivos para o setor automobilístico levaram a um boom no mercado de crédito dessa modalidade que apresentou crescimento de $49 \%$ entre 2009 e 2010, atingindo $\mathrm{R} \$ 149$ bilhões em março de 2011 (13,0\% do crédito livre). Contudo, este boom foi seguido de um aumento na inadimplência desde o final de 2010. A inadimplência do financiamento para veículos da pessoa física chegou a um patamar histórico, alcançando 5,5\% em fevereiro de 2012. Em fevereiro de 2010, a fim de reduzir os riscos dessa expansão de crédito, foram editadas as chamadas medidas macroprudenciais para recompor o montante de recolhimentos compulsórios sobre os depósitos a níveis pré-crise crédito que objetivaram basicamente a redução dos prazos do crédito pessoal e do financiamento de veículos para pessoas físicas, além do termino da redução do IPI.

No contexto supracitado, este artigo apresenta estimativas da função de demanda de crédito para veículos no Brasil com dados agregados para o período entre junho de 2000 a dezembro de 2012. Pode-se observar nesse período a existência de períodos de expansão seguidos de fase de retração. Nesse sentido, procura-se construir uma história capaz de explicar o porquê desses ciclos. Com base nas estimativas da demanda por crédito avalia-se possíveis mudanças de regimes que podem ter ocorrido em função das mudanças de marcos legais no setor creditício, de medidas anticíclicas adotadas pelo governo em decorrência de crises econômicas e também devido a mudanças de orientação de política econômica, a exemplo da chamada "Nova Matriz Macroeconômica". 
Após esta breve introdução, a seção um faz uma descrição das principais mudanças institucionais ocorridas, sobretudo, no mercado de crédito para veículos assim como descreve as medidas anticíclicas adotadas pelo governo para o setor automobilístico. Naturalmente os dados agregados de concessão de crédito refletem o equilíbrio entre a demanda e oferta de financiamento. De modo a identificar a função de demanda usamos duas estratégias complementares. Na seção 2, introduzimos o modelo para a função demanda por crédito para veículo usando métodos tradicionais de identificação como IV/2SLS e GMM. A seguir, tendo em vista os impactos sofridos no segmento de crédito para veículos em decorrência das medidas já assinaladas, usamos um modelo Markov Switching (MS) com variável endógena (Kim, 2004) de modo a captar os diversos regimes a que esteve sujeita a função de demanda por concessão de crédito no segmento de veículos, seguindo-se os comentários finais.

Nossos resultados mostraram que foi possível identificar a função de demanda por financiamento de veículos na qual o preço do veículo, a taxa e prazo de financiamento assim como o estado da economia são as variáveis explicativas do modelo, ou seja, a função de demanda por concessão de crédito para veículo segue de perto o que se espera de uma função de demanda tradicional. Por outro lado, o modelo MS mostrou que tal função de demanda passou por três regimes distintos ao longo do período amostral. Aqui temos um regime de expansão e retração que são inerentes a uma economia de mercado. Destaca-se ainda a existência de um terceiro regime que também é de expansão só que gerado de modo artificial em decorrência do emprego de medidas anticíclicas fomentadas pelo governo.

\section{Concessão de crédito para veículos: fatos estilizados}

O Gráfico 1 apresenta a série histórica de concessão de crédito para veículo no Brasil de junho de 2000 a dezembro de 2012. Nela, se observa a existência de períodos de expansão seguidos de fase de retração. A tarefa, nesta seção, consiste em construir uma história capaz de explicar o porquê desses ciclos. Com o objetivo de facilitar a análise, foram inseridas três linhas e duas faixas sombreadas. O intervalo entre as duas primeiras linhas vai de meados de 2004 até o início de 2008, enquanto a terceira linha marca 
o início de 2011. Observa-se que o intervalo entre agosto de 2004 e início de 2008 está associado a uma fase longa de expansão do ciclo de crédito. Nota-se, ainda, que, durante o ano de 2008, a série de concessão de crédito apresentou um período de queda muito acentuada, seguida, logo após, de movimento expressivo de retomada de crescimento até o final do ano de 2010. A partir do início de 2011 parece haver uma tendência de queda. As faixas sombreadas indicam redução das alíquotas do IPI.

\section{Gráfico 1}

Concessão de crédito: veículos

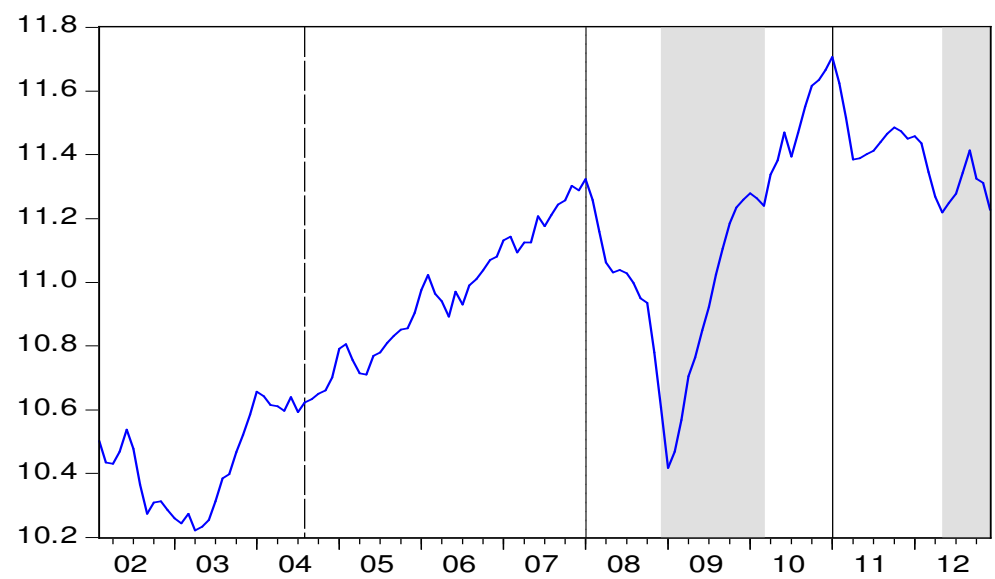

Fonte: Elaboração dos autores.

A hipótese, aqui, é que o primeiro ciclo de expansão foi impulsionado por fatores pró-mercado, tais como as mudanças advindas da legislação, a queda na taxa de juros, o crescimento da economia, o aumento da renda real etc. Foi visto na seção anterior que o mercado de crédito experimentou várias mudanças institucionais importantes até meados da década de 2000, entre as quais se destacam o instituto da alienação fiduciária ${ }^{5}$. Assunção, Benmelech e Silva (2012) mostram que houve mudanças estruturais no perfil do crédito para aquisição de veículos em decorrência da introdução da lei da alienação fiduciária em agosto de 2004,

(5) A lei da alienação fiduciária não se restringiu apenas ao mercado de veículos. Teve efeito importante também no setor imobiliário. 
como a diminuição do spread, o aumento dos empréstimos e do prazo de financiamento ${ }^{6}$. Deve-se ter em mente que a quebra estrutural vista na série de concessão de financiamento para veículo aconteceu em outros segmentos importantes do mercado de crédito $^{7}$, dando a entender, naturalmente, que essa mudança deve estar ligada fortemente às causas estruturais já mencionadas ou aos fundamentos da economia.

A partir de janeiro de 2008, verifica-se uma queda acentuada nas concessões de financiamento para veículos, o que está diretamente associado à eclosão da crise financeira mundial ocorrida entre 2008-2009 ${ }^{8}$. De modo a contrabalançar uma eventual falta de liquidez no sistema bancário, o BCB, em outubro de 2008, optou pela diminuição dos recolhimentos compulsórios sobre depósitos para suprir as necessidades dos bancos menores 9 (Martins, Lundberg, Takeda, 2008). Não obstante, o governo usou outras medidas para estimular o mercado de veículos que tiveram imediata repercussão no mercado de crédito, como a diminuição da alíquota do Imposto Sobre Produtos Industrializados (IPI) que vigorou entre dezembro de 2008 a fevereiro de 2010 . O período em que vigora a redução do IPI é representado pela primeira faixa escura do Gráfico 1. Provavelmente, ambas as medidas, o aumento da liquidez assim como a queda no preço dos veículos, decorrente da redução do IPI, tenham contribuído para o aumento da demanda por automóveis ${ }^{10}$, levando a uma retomada expressiva da expansão do crédito já no início de 2009. Essa

(6) No entanto, diferentemente deste último, onde o colateral, no caso o veículo, se desvaloriza substancialmente logo após a venda e mantém esta tendência, o imóvel, em geral, tende a se valorizar ou pelo menos acompanhar a inflação. Assim, depreende-se que o efeito do instituto da alienação fiduciária tenha sido bem maior no mercado imobiliário que em outros mercados.

(7) Para uma análise da evolução do crédito imobiliário, ver Mendonça e Sachsida (2013)

(8) Martins (2010) analisa os efeitos sobre a restrição de crédito decorrentes da crise de 2008.

(9) Em virtude da concentração do sistema bancário e da estrutura progressiva dos recolhimentos compulsórios, a maior parte dessas reservas encontra-se vinculada aos bancos de grande porte, que sofreram relativamente menores restrições de liquidez. Então, no conjunto de medidas adotadas para sanar os problemas de distribuição de liquidez, vinculou-se parte da liberação dos compulsórios dos bancos de grande porte ao direcionamento de parte desses recursos aos bancos menores, principalmente por meio de compra de suas carteiras de crédito ou aplicação em DIs.

(10) Para uma análise do impacto da redução do IPI sobre as vendas de automóveis, ver Alvarenga et al. (2010). 
retomada persistiu até mesmo ao término do IPI reduzido que voltou ao nível anterior em março de 2010.

Contribui também para a retomada do crédito a queda da taxa básica de juros Sistema Especial de Liquidação e de Custódia (Selic), que permitiu a diminuição na taxa de financiamento. Também foram facilitadas as condições para liberação de crédito. Nesta época, conseguia-se comprar um automóvel sem entrada e com prazo de financiamento de até 90 meses. Durante este período houve aumento do endividamento, o que levou o BCB, a partir de fevereiro de 2010, com o fim de reduzir os riscos da expansão do crédito, editar as chamadas medidas macroprudenciais, de modo a recompor o montante de recolhimentos compulsórios sobre os depósitos a níveis précrise. Em dezembro de 2010, outras medidas de elevação dos recolhimentos compulsórios foram implantadas. Vieram acompanhadas de medidas específicas para a moderação das concessões de crédito, que objetivaram basicamente a redução do prazo do financiamento de veículos para pessoas físicas, por meio da majoração do requerimento de capital para as concessões de crédito de prazo acima de 24 meses $^{11}$.

Apesar da introdução das medidas macroprudenciais, a inadimplência ${ }^{12}$ de pessoas físicas na aquisição de veículos começou a crescer continuamente desde dezembro de 2010, quando estava em 2,5\%. Em maio de 2011, o saldo de inadimplência já era de 3,6\%. E em maio de 2012, a inadimplência acima de 90 dias atingiu 6,1\%. Boa parte da crise no financiamento de veículos decorreu de vendas realizadas entre 2010 e 2011 , quando as taxas de juros eram menores e os prazos de pagamentos mais longos, como pode ser visualizado no Gráfico 2. Conjugadas com as condições macroeconômicas, todas essas medidas levaram novamente a retração da atividade creditícia no segmento de veículos, que teve início em

(11) O BCB teve como objetivo reduzir os recursos para financiamentos com prazo superior a 24 meses. Pela nova regra, para não cair na restrição, os bancos tiveram que exigir uma entrada de pelo menos $20 \%$ nos financiamentos entre 24 e 36 meses para carros novos ou usados. Nos parcelamentos entre 36 e 48 meses, a entrada subiu para $30 \%$ e, entre 48 e 60 meses, para $40 \%$. Vendas de veículos com prazo superior a 60 meses tiveram restrição, independentemente da entrada. Para limitar essas operações, o BCB exigiu que os bancos criassem uma reserva de capital 50\% maior para garantir esses empréstimos.

(12) Inadimplência acima de 90 dias. 
janeiro de 2011 como mostra o Gráfico 1. De modo a incentivar a indústria automobilística, o governo novamente promoveu, a partir de maio de 2012, a diminuição da alíquota do IPI, segunda área sombreada do Gráfico 1. Isso parece ter tido certo efeito positivo, pois, como pode ser visto no gráfico a seguir, houve um aumento das concessões embora a situação tenha se revertido alguns meses à frente.

\section{Gráfico 2}

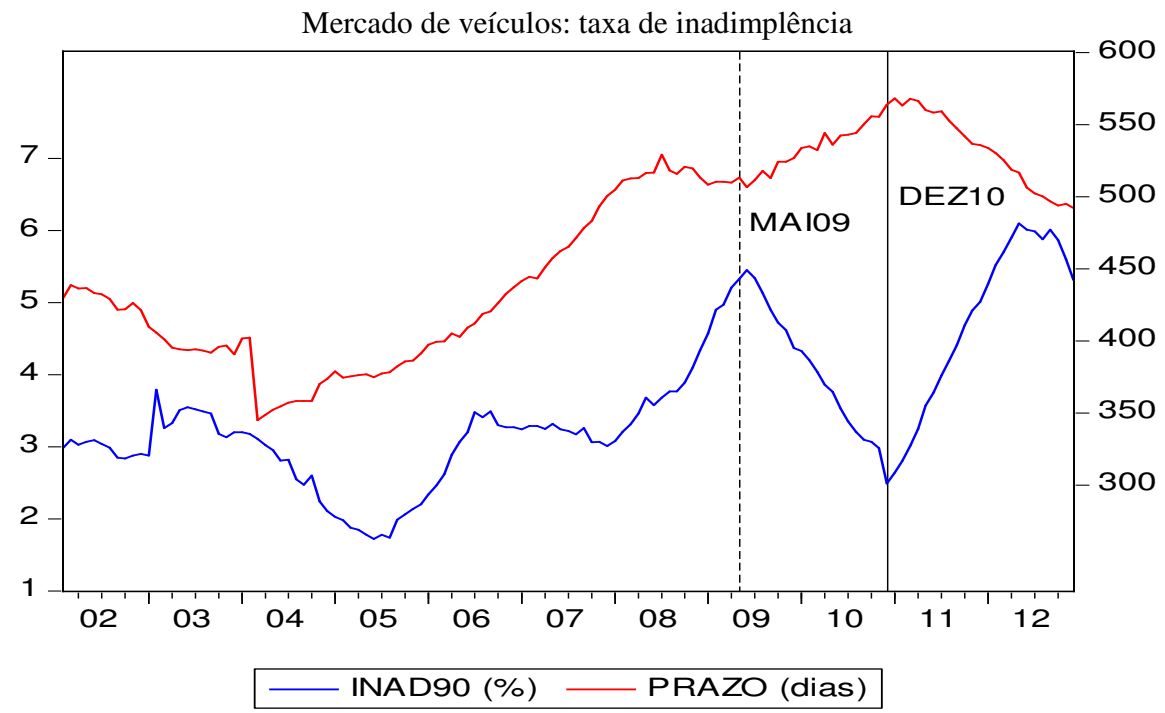

Fonte: Elaboração dos autores.

\section{Função de demanda de crédito para veículo}

Conforme foi visto na seção anterior existiram desde 2004 vários fatores que afetaram a concessão de crédito para veículos. Tais fatores certamente tiveram importantes efeitos tanto sobre o lado da oferta de crédito assim como sobre o lado da demanda. O Gráfico 1 pode ser naturalmente interpretado como um conjunto de pontos representativo da interação entre as curvas de demanda e de oferta de concessão de crédito dispersos temporalmente. Isso se assemelha às variáveis quantidade e preço da interação entre as funções de demanda e oferta, tópico muito comum nos 
livros de econometria. A questão que tratamos nesta seção diz respeito à identificação da função de demanda de concessão de crédito. Nossa proposta recai numa especificação para a função de demanda de concessão de crédito que assume a seguinte forma

$$
\mathrm{CONCVC}_{t}=b_{0}+b_{1} \operatorname{TAXA}_{t}^{*}+b_{2} \operatorname{PREVCN}_{t}+b_{3} \underset{+}{\text { PRAZO }_{t}+b_{4} \operatorname{TXPIB}_{t}+\varepsilon_{t}}
$$

onde temos que:

CONCVC: concessões de crédito para financiamento de veículos, livremente compactuadas entre mutuários e instituições financeiras acumuladas no mês. Fonte: BCB;

TAXA: Taxa média mensal anualizada das operações de financiamento de veículos para pessoa física com recursos livres: Fonte: $\mathrm{BCB}$;

TXPIB: Taxa crescimento do PIB real acumulado nos últimos 12 meses. Fonte: BCB;

PRAZO: Prazo médio em dias dos contratos de financiamento de veículos. Fonte: $\mathrm{BCB}$; e

PREVCN: Índice de Preços de Veículos Novos. Fonte: IBGE.

As variáveis CONCVC e TAXA são as variáveis endógenas do modelo. Incluímos ainda como variáveis exógenas o índice de preço de veículos novos (PREVCN), pelo prazo de financiamento $(P R A Z O)$ e a taxa de crescimento real do PIB (TXPIB) que tem a função de uma variável proxy para a atividade econômica. Os sinais esperados para os coeficientes das variáveis aparecem abaixo de cada uma delas. No caso da variável TAXA o sinal esperado é negativo. Ela ainda aparece com um asterisco que indica possível problema de endogeneidade. A exceção dessa variável todas as demais são assumidas como exógenas com sinal esperado positivo. Deve-se assinalar que o uso preço do veículo novo permite captar de modo direto o efeito das mudanças na alíquota do IPI ocorridas a partir do início de 2008.

Ante o exposto, a demanda por crédito é caracterizada por uma relação inversamente proporcional com a taxa de financiamento dos 
automóveis. Nesse caso, quando os agentes econômicos adiam o consumo presente para gerar poupança para financiar os demandantes de crédito ele espera uma recompensa no futuro, que seria o "preço" pelo seu sacrifício de renunciar o consumo presente, ou seja, a taxa de juros cobrada no financiamento dos veículos novos. Assim, menores taxas implicam em maior demanda por crédito. Além disso, a demanda por crédito responde positivamente a maiores prazos e variações positivas da renda. Essas 3 variáveis explicativas é que caracterizam uma função demanda por crédito tradicional. Isso posto, ainda estamos utilizando uma variável que pretende capturar o efeito da redução do IPI sobre a compra de automóveis, que é o índice de preços dos veículos novos. Admita que um cliente pretenda comprar um veículo novo com um valor fixo de entrada e o restante financiado. No momento da negociação o vendedor diz que o governo reduziu a alíquota do IPI e que o preço do automóvel reduz no mesmo valor da redução do imposto ${ }^{13}$. Dessa forma, o comprador vai reduzir o montante do crédito no valor equivalente à redução do IPI, o que significa que há uma relação diretamente proporcional ou positiva entre demanda por Crédito e preço do automóvel novo.

No exemplo supracitado, uma redução do preço do automóvel devido à redução da alíquota do IPI, resultou numa redução do montante do credito. Da mesma forma, um aumento do IPI sobre os veículos novos vai aumentar o preço do veículo e acarretar um maior montante de crédito para arcar com os encargos fiscais. Assim, espera-se uma relação direta entre demanda por credito e preços de veículos novos.

\subsection{Estratégia de identificação}

A estratégia de identificação a ser empregada segue os seguintes passos. Primeiro, qualquer que seja o método usado para lidar como problema da endogeineidade (IV, 2SLS, GMM, etc.), deve-se postular um conjunto de variáveis instrumentais de modo a servirem de exógenas na forma reduzida da equação que modela a variável TAXA. Usamos aqui a taxa

(13) Não necessariamente a redução tem que ser na mesma proporção, o que não invalida o argumento. 
de inadimplência acima de 90 dias na modalidade de crédito referida e taxa SELIC, taxa básica de juros da economia, como instrumentos da variável TAXA. Assim a forma reduzida assume o seguinte formato,

$$
\begin{gathered}
T A X A_{t}=\delta_{0}+\delta_{1} \operatorname{SELIC}_{t}+\delta_{2}{ }_{+}^{I N A D 90} t \\
+
\end{gathered}
$$

Por economia não incluímos em (2) as demais variáveis exógenas que aparecem na equação (1). Contudo isso é naturalmente feito quando do procedimento de estimação por 2SLS/IV. Em segundo lugar, aplicaremos os testes econométricos usuais de modo a nos certificarmos se problema de endogeneidade (sub-identificação, sobre-identificação Hausman) de fato acontece. A seguir, estimaremos a função de demanda com base no método de dois estágios (2SLS/IV) e aplicamos os testes de especificação (heteroscedasticidade, autocorrelação serial, instrumentos fracos, etc.) na regressão estimada. Por fim, estimamos a função de demanda corrigindo os erros de especificação.

Para que a aplicação do método IV/2SLS seja adequada se faz necessário que os instrumentos sejam "bons instrumentos" no sentido de serem relevantes bem como válidos. No jargão econométrico isso significa que as variáveis usadas como instrumentos devam ser correlacionadas com os regressores endógenos e ao mesmo tempo ortogonais ao distúrbio da regressão. Neste sentido são aplicados o teste de sub-identificação ${ }^{14}$ (CraggDonald, 1993), Kleibergen na Paap (2006) ${ }^{15}$, o teste de sobre-identificação

(14) Para que uma equação seja identificada no modelo IV tanto a condição de ordem (L>=K), onde $\mathrm{L}$ é o número de variáveis instrumentais e $\mathrm{K}$ o número de regressores, como a condição de posto devem ser preenchidas. Esta última assinala que $\mathrm{Qxz}=\mathrm{E}\left(\mathrm{X}^{\prime} \mathrm{Z}\right)$ deve ser de posto completo, onde $X=(X 1, X 2)=($ Endógenas, Exógenas) é a matriz de regressores, enquanto $Z=(Z 1, Z 2)=$ (Exclusos, Inclusos) é a matriz dos instrumentos, sendo $Z 2=X 2$. Quando isso não ocorre dizemos que o modelo é sub-identificado ou não identificado. Pode-se testar a condição de posto por meio do teste de CraggDonald (1993), cuja não rejeição da hipótese nula sugere que o modelo é sub-identificado.

(15) $\mathrm{Na}$ presença de heteroscedasticidade e autocorrelação residual, a estatística de CraggDonald não mais permanece válida. Em tais circunstâncias, o teste de Kleibergen na Paap (2006) será usado como alternativa ao teste Cragg-Donald (1993) quando as circunstâncias assim exigirem. 
de Sargan-Hansen ${ }^{16}$, além do teste de Stock-Yogo (Stock; Yogo, 2005) para verificar se os instrumentos são fracos ${ }^{17}$.

A pesquisa teórica econométrica mais recente sobre o método de variáveis intrumentais tem enfatizado muito a questão dos instrumentos fracos (Stock; Wright; Yogo), (Moreira, 2003), (Stock; Yogo, 2005). Quando os instrumentos são fracos, dois problemas sérios ocorrem na estimação por dois estágios (2SLS). O primeiro é a questão do viés. Embora o método 2SLS seja consistente ${ }^{18}$, as estimativas são sempre viesadas para pequenas amostras. Segundo, quando os instrumentos são fracos, o erro padrão estimado se torna muito pequeno. Neste caso, o intervalo de confiança é não fidedigno, pois juntamente ao fato de que o ponto médio deste estimador ser viesado, temos que o intervalo de confiança se torna pequeno. Isso tudo faz com que o procedimento de teste de hipótese na estimação por 2SLS se fragilize no caso da ocorrência de instrumentos fracos. Como Staiger e Stock mostraram o problema de instrumentos fracos pode ocorrer mesmo se no primeiro estágio os testes são significativos nos níveis convencionais (5\% ou 10\%) e para grandes amostras.

Vários testes são sugeridos na literatura para testar a hipótese de instrumentos fracos. Uma estatística comumente usada é o $\mathrm{R}^{2}$ do primeiro estágio com os instrumentos dito "inclusos" (Bound et al., 1995) ${ }^{19}$. Contudo,

(16) A independência do instrumento com relação ao distúrbio somente pode ser acessada se, e somente se, houver uma "abundância" de instrumentos, isto é, se a equação é sobre-identificada. Isto se dá quando a condição de ordem é satisfeita na desigualdade: o número de instrumentos excluídos é superior ao regressores endógenos. O teste de Sargan/Hansen é usado para testar a hipótese de sobreidentificação. Sob a hipótese nula os instrumentos são válidos, ou seja, não correlacionados com o distúrbio. Sob ainda esta hipótese, a estatística de teste tem distribuição qui-quadrado com L-K restrições sobre-identificadas.

(17) Instrumentos que explicam pouco a variação da variável explicativa endógena (fraca correlação entre Z e X) são considerados instrumentos fracos. O teste de Stock-Yogo (Stock-Yogo, 2005) é calculado com base na estatística F de Cragg-Donald (1993). Sob a hipótese nula o estimador é fracamente identificado no sentido de que o viés verificado é inaceitavelmente grande.

(18) De modo que quase certamente o valor médio do estimador 2SLS se aproxima do verdadeiro valor do parâmetro quando o tamanho da amostra convirja para o infinito.

(19) Alternativamente, isso também pode ser expresso como um teste $\mathrm{F}$ da significância conjunta dos instrumentos exclusos Z1. 
quando existe mais de uma variável endógena na regressão, esse indicador pode não ser mais válido. Shea (1997) propôs uma estatística conhecida como " $\mathrm{R}^{2}$ parcial" que capta a intercorrelação entre os instrumentos. Quando somente existe uma variável endógena, as duas medidas de $\mathrm{R}^{2}$ são equivalentes. Uma outra regra aplicada quando se tem apenas um regressor endógeno é checar se o valor da estatística $\mathrm{F}$ no primeiro estágio. Nesse caso, um valor menor que 10 pode ser uma indicação que os instrumentos são fracos. Alternativamente Stock and Yogo (2005) sugerem um teste onde a hipótese nula é que o viés do estimador 2SLS seja menor que uma fração (digamos, 10\%) do estimador OLS ${ }^{20}$. De acordo com Murray (2006) a mais recente abordagem em relação ao problema de teste de hipótese com instrumentos fracos e um único regressor endógeno é o "teste da razão de verossimilhança condicional", desenvolvido por Moreira (2003) e aperfeiçoado em Andrews, Moreira e Stock (2006) e Andrews e Stock (2005). O teste de Moreira (2003) supera as distorções encontradas nos testes convencionais ajustando os valores críticos do teste de hipótese de acordo com cada amostra, de modo que o intervalo de confiança corrigido gere um nível de significância correto. Assim, seus valores críticos "são condicionados" nos dados à disposição e não constante.

Embora muitos estudos utilizem a abordagem GMM para estimação de modelos com problemas de endogeneidade, deve-se assinalar que esta metodologia não necessariamente é superior à estimação feita pelo método IV/2SLS. Tal como ressalta Baum et al (2007) numa excelente resenha acerca da aplicação dos métodos IV e GMM, a vantagem da abordagem GMM se dá sobretudo na presença de heteroscedasticidade ${ }^{21}$. Ainda assim, tal vantagem em termos da propriedade de consistência do estimador GMM na presença de heteroscedasticidade pode ser ofuscada pelo custo de uma performance fraca para o caso de pequenas amostras. De modo a checar a

(20) O teste de Stock-Yogo (Stock e Yogo, 2005) não mais recai na estatística $F$ que comumente aparece na prática econométrica, mas numa generalização da estatística de Cragg-Donald. Os valores críticos deste teste são obtidos a partir de uma distribuição não padrão.

(21) Embora na presença de heterocedasticidade a propriedade de consistência dos coeficientes estimados pelo método IV seja mantida, na estimação básica de variáveis instrumentais os erros padrão são inconsistentes e, portanto a inferência fica comprometida (Baum et al., 2007). 
existência de heteroscedasticidade na regressão, aplicamos neste estudo o uma versão específica do teste de Pagan e Hall (1983) ${ }^{22}$.

\subsection{Resultados}

A Tabela 1 apresenta os resultados obtidos a partir dos procedimentos econométricos apresentados na seção anterior. Emprega-se dados agregados de junho de 2000 a dezembro de 2012. A coluna (1) usa o estimador simples de mínimos quadrados ordinários (OLS) visando estimar a função de demanda por concessão de crédito para veículos. Esse método não corrige o possível problema de endogeneidade levantado acima, mas nos permite aplicar a estatística VIF $^{23}$ de modo a inferir por meio dela se existe problema de multicolinearidade na regressão (Lutkepohl, 1983). A coluna (2) apresenta o resultado da estimação da forma reduzida da equação (2) referente à variável TAXA. Naturalmente além dos instrumentos excluídos Selic e INAD90, a regressão é estimada incorporando as demais variáveis exógenas do modelo. Observa que os sinais das variáveis SELIC e INAD90 são compatíveis com os esperados. As colunas (3) e (4) estimam a regressão para a variável CONCVCN corrigindo o viés de endogeneidade. A regressão apresentada na coluna (3) é estimada pelo método OLS e inclui entre os regressores a variável $\operatorname{ERRO}(2)$ que é o resíduo obtido da regressão na coluna (2). Essa regressão nos permite visualizar a existência de endogeneidade na variável TAXA ${ }^{24}$. Note o coeficiente dessa variável é significativo indicando que o problema de endogenia na regressão da coluna (1) se mostra factível.

Na coluna (4) estima-se a função de demanda por concessão de crédito pela aplicação do método de dois estágios (2SLS). Percebe-se que os resultados das colunas (3) e (4) são muito próximos. Com base na regressão da coluna (4) aplicamos os testes de especificação descritos na seção anterior. Para verificar se os instrumentos são fracos temos o $R^{2}$ que

(22) Este teste assume a hipótese nula de homocedasticidade.

(23) Variation Inflation Factor. Um valor abaixo de 5.00 indica a não ocorrência de problema de multicolinearidade.

(24) Para maiores detalhes acerca deste procedimento ver Wooldridge (2006). 
aparece na coluna (2) do primeiro estágio com os instrumentos dito "inclusos" (Bound et al., 1995) além do teste Stock-Yogo. De acordo com essas duas estatísticas as variáveis instrumentais utilizadas não se configuram num conjunto de instrumentos fracos. Vejamos se as variáveis instrumentais atendem a condição de serem "bons instrumentos". O teste de sub-identificação indica que a condição de ordem é preenchida enquanto o teste de Sargan/Hansen não rejeita a hipótese nula de que os instrumentos excluídos sejam não correlacionados com o resíduo. Por fim, os testes de Pagan-Hall e Cumby-Huizinga são aplicados visando verificar a existência de, respectivamente, heteroscedasticidade e de autocorrelação. Os resultados mostram que tais problemas de especificação devem ser levados em conta. De modo a corrigir isso, empregamos na coluna (5) o método GMM considerando os procedimentos apropriados para correção de tais problemas de especificação.

Em relação aos resultados obtidos, é oportuno mencionar que os sinais encontrados e níveis de significância estão em conformidade com o esperado. Para cada variável da equação (1) os respectivos sinais dos coeficientes de todas as regressões são os mesmos. A demanda concessão de financiamento reage negativamente à taxa de financiamento, positivamente com relação ao prazo de financiamento, o preço do carro novo e a taxa de crescimento do PIB. Conforme já foi colocado, essa variável tem como função captar o estado da atividade econômica. Neste último caso também usamos a taxa de desemprego (DESP) que serve como variável de controle refletindo o estado da economia. Tendo em vista o fato que o sinal obtido quando se usa a variável DESP na equação (1) é negativo, os sinais das demais variáveis se mantiveram quantitativa e qualitativamente inalterados ${ }^{25}$.

(25) Taxa de desemprego aberto das regiões metropolitanas. Fonte: Pesquisa Mensal de Emprego (PME). Fonte: Instituto Brasileiro de Geografia e Estatística (IBGE). Por economia não apresentamos os resultados. No caso de haver interesse entrar em contato com os autores. 
Uma avaliação da demanda creditícia para automóveis no Brasil no período de 2000 a 2012

Tabela 1

Equação de Demanda de Crédito

\begin{tabular}{|c|c|c|c|c|c|}
\hline & $\begin{array}{l}\text { OLS } \\
(1)\end{array}$ & $\begin{array}{c}\text { OLS } \\
(2)\end{array}$ & OLS & 2SLS/IV* & $\mathrm{GMM}^{*}$ \\
\hline & $\begin{array}{c}\text { LCONCVC } \\
\text { (1) }\end{array}$ & $\begin{array}{l}\text { TAXA } \\
(2)\end{array}$ & $\begin{array}{c}\text { LCONCVC } \\
\text { (3) }\end{array}$ & $\begin{array}{c}\text { LCONCVC } \\
\text { (4) }\end{array}$ & $\begin{array}{c}\text { LCONCVC } \\
(5)\end{array}$ \\
\hline TAXA & $\begin{array}{c}-0.026 \\
(0.000) \\
\end{array}$ & & $\begin{array}{l}-0.022 \\
(0.000)\end{array}$ & $\begin{array}{l}-0.022 \\
(0.000)\end{array}$ & $\begin{array}{l}-0.212 \\
(0.000) \\
\end{array}$ \\
\hline PRAZO & $\begin{array}{c}0.013 \\
(0.000)\end{array}$ & $\begin{array}{c}0.013 \\
(0.000)\end{array}$ & $\begin{array}{c}0.001 \\
(0.0003)\end{array}$ & $\begin{array}{c}0.001 \\
(0.000)\end{array}$ & $\begin{array}{c}0.001 \\
(0.023)\end{array}$ \\
\hline TXPIB & $\begin{array}{c}0.006 \\
(0.003)\end{array}$ & $\begin{array}{c}0.432 \\
(0.034)\end{array}$ & $\begin{array}{c}0.005 \\
(0.025)\end{array}$ & $\begin{array}{c}0.054 \\
(0.416)\end{array}$ & $\begin{array}{c}0.008 \\
(0.163)\end{array}$ \\
\hline LPRVECN & $\begin{array}{c}0.008 \\
(0.000) \\
\end{array}$ & $\begin{array}{c}0.138 \\
(0.017) \\
\end{array}$ & $\begin{array}{c}0.008 \\
(0.000) \\
\end{array}$ & $\begin{array}{c}0.008 \\
(0.000) \\
\end{array}$ & $\begin{array}{c}0.009 \\
(0.000) \\
\end{array}$ \\
\hline INAD90 & & $\begin{array}{c}0.936 \\
(0.000)\end{array}$ & & & \\
\hline SELIC & & $\begin{array}{c}1.698 \\
(0.000) \\
\end{array}$ & & & \\
\hline $\operatorname{ERRO}(2)$ & & & $\begin{array}{l}-0.014 \\
(0.031)\end{array}$ & & \\
\hline CTE & $\begin{array}{l}-13.155 \\
(0.120) \\
\end{array}$ & $\begin{array}{l}-13.155 \\
(0.120) \\
\end{array}$ & $\begin{array}{l}11.076 \\
(0.000)\end{array}$ & $\begin{array}{c}10.67 \\
(0.000) \\
\end{array}$ & $\begin{array}{l}10.497 \\
(0.000)\end{array}$ \\
\hline OBS & 151 & 151 & 151 & 151 & 151 \\
\hline VIF & 2.17 & & & & \\
\hline $\begin{array}{c}\text { Teste de } \\
\text { Pagan-Hall }\end{array}$ & & & & $\begin{array}{l}20.442 \\
(0.002)\end{array}$ & \\
\hline $\begin{array}{c}\text { Teste Cumby- } \\
\text { Huizinga }\end{array}$ & & & & $\begin{array}{c}66.53 \\
(0.000)\end{array}$ & \\
\hline $\begin{array}{l}\text { Teste de sub- } \\
\text { identificação }\end{array}$ & & & & $\begin{array}{c}96.38 \\
(0.000) \\
\end{array}$ & $\begin{array}{l}17.039 \\
(0.000) \\
\end{array}$ \\
\hline $\begin{array}{c}\text { Teste de } \\
\text { Sargan/Hansen }\end{array}$ & & & & $\begin{array}{l}18.867 \\
(0.001) \\
\end{array}$ & $\begin{array}{c}2.04 \\
(0.031) \\
\end{array}$ \\
\hline $\mathrm{R}^{2} \mathrm{Adj}$ & 82.33 & 87.75 & & & \\
\hline $\begin{array}{c}\text { Teste de } \\
\text { Stock-Yogo } \\
(5 \%)\end{array}$ & & & & $\begin{array}{c}84.072 \\
13.91\end{array}$ & $\begin{array}{c}84.072 \\
0.013\end{array}$ \\
\hline
\end{tabular}

Fonte: Elaboração dos autores. Nota: P-valor entre parênteses. L: transformação logarítmica. 


\subsection{Demanda de crédito para veículos por mudança de regime}

Conforme foi visto na seção introdutória, o mercado de concessão de crédito para veículos sofreu efeitos importantes decorrentes de diversos fatores. Tais impactos decorreram de mudanças incidentes não somente sobre a regulação geral do crédito assim como aquelas referentes à regulação específica para a concessão de crédito para veículos. Além disso, também vimos que o governo procurou incentivar o setor automobilístico reduzindo durante certos períodos a alíquota do IPI sobre veículos. É razoável se supor que tais alterações tenham conduzido à ocorrência de quebras estruturais na série de concessões de financiamento.

Nosso objetivo nesta seção é revisitar o problema acerca da estimação da função de demanda por concessão de crédito para veículos considerando-se as supostas quebras estruturais por meio de um procedimento adequado, o que não foi possível de fazer na seção 3. Um problema potencialmente importante quando existe intervenção ou mudança de regime é o fato das técnicas tradicionais não serem robustas à existência de quebras estruturais nos dados (Leybourne; Newbold, 2003; Noh; Kim, 2003; Cook, 2004), quebras essas que certamente ocorreram na concessão de crédito. Uma alternativa empregada para tratar quebras estruturais (e, portanto, as "mudanças de regime") exógenas se faz por meio da introdução de variáveis dummies em modelos lineares convencionais. Contudo, tal procedimento exige que se conheça antecipadamente o momento exato onde ocorreram as quebras, o que raramente é o caso na prática. E mesmo no caso improvável de o pesquisador "acertar" a data exata da(s) quebra(s) relevante(s) bem como, respectivamente os períodos de duração das quebras, a mera introdução de dummies não resolve problemas relacionados a mudanças de regime na variância dos erros do modelo.

Quando uma relação linear é submetida a uma quebra estrutural, ${ }^{26}$ os parâmetros do modelo de regressão variam com tempo, resultando em não linearidades e, via de regra, em violações das hipóteses de estacionariedade e normalidade dos erros dos modelos convencionais.

(26) O que pode ocorrer nos coeficientes das variáveis, no intercepto e também na variância dessa relação. 
Como aponta Sims (2001), é um equívoco grave ignorar quaisquer fontes de não normalidade nos resíduos ao mesmo tempo em que se leva em conta mudanças nos parâmetros das variáveis. De fato, o teste de Pesaran and Taylor (1999) por vezes denominado de teste de variável omitida, mas provavelmente melhor interpretado como sendo um teste para checar a ocorrência de não linearidades na forma funcional (Wooldridge 2002, p. 124-125), quando aplicado ao modelo da equação (1) estimado por OLS, rejeita a hipótese nula de não ocorrência de não linearidades negligenciadas ${ }^{27}$.

Neste estudo, a proposta é lidar com as referidas quebras estruturais por meio do emprego de um modelo que admite explicitamente a existência de vários regimes nos dados (denominado Markov-Switching Models), estimando as probabilidades de transição entre esses diferentes regimes endogenamente através do uso das chamadas Cadeias de Markov (Hamilton, 1989, 1994; Krolzig, 1997 e Sims, 1999, 2001).

Modelos do tipo $\mathrm{MS}^{28}$ se caracterizam por assumir explicitamente a possibilidade de que, a cada momento do tempo, um número finito (e geralmente pequeno) de "regimes" ou "estados" pode ocorrer, sem que se saiba ao certo qual deles está sendo observado. A hipótese é que existem "probabilidades de transição" de um regime para o outro, probabilidades essas estimadas endogenamente pelos modelos $\mathrm{MS}^{29}$. Nada impede, ainda, que as mudanças de regime sejam aquelas do tipo once-and-for-all-shifts,

(27) Sob a hipótese nula, não há não linearidades negligenciadas, os resíduos devem ser não correlacionados com os polinômios de baixa ordem dos valores projetados da variável dependente. O teste estatístico (Wald ou distância GMM) para pequenas (estatística F) ou grandes amostras (Chi-2) com um número de graus de liberdade igual os número de termos polinomiais $[(\mathrm{F}(1,110)=5.04-P$ value $=0.0268),($ Chi-sq $(1)=5.36, P$-value $=0.0206)]$.

(28) Mais tecnicamente, modelos MS se enquadram naquilo que se Chib (1996) denomina de "hidden Markov chain models". Uma ampla variedade desses modelos é apresentada em Kim e Nelson (1999).

(29) Apenas para citar um exemplo intuitivo, parece razoável supor que uma economia em recessão se comporte de modo (ou tenha parâmetros) diferente(s) de uma economia em rápido crescimento. Nesse caso, pode-se pensar em dois "regimes", isto é, um "recessivo" e outro "de crescimento", com características bastante diferentes entre si e que se alternam de tempos em tempos, sem que se tenha certeza sobre qual está ocorrendo em cada período específico. 
nas quais, após a mudança, o novo regime permanece indefinidamente. Tendo em vista os comentários feitos até aqui, a proposta deste estudo é estimar a equação de demanda por concessões de crédito para veículos, com base em um modelo MS. Daí a equação (1) pode ser reescrita numa versão para mudança de regime tipo MS da seguinte forma

$$
\operatorname{CONVC}_{t}=b_{0}\left(s_{t}\right)+b_{1}\left(s_{t}\right){ }_{-} \operatorname{TAXA}_{t}^{*}+b_{2}\left(s_{t}\right) \underset{+}{\operatorname{PREVCN}_{t}+b_{3}\left(s_{t}\right) \underset{+}{P A Z O_{t}}+b_{4}\left(s_{t}\right){ }_{-}^{T X P I B} B_{t}+\varepsilon_{t}}
$$

onde $\varepsilon_{t} \sim N\left(0, \sigma^{2}\left(s_{t}\right)\right)$;

Note-se que, por hipótese, a "variável latente" $s_{t}$ é regida por um processo estocástico conhecido como uma cadeia de Markov ergódica e definido por uma matriz de probabilidades de transição cujos elementos são dados por:

$$
\begin{aligned}
& p_{i j}=\operatorname{Pr}\left(s_{t+1}=j \mid s_{t}=i\right), \quad \sum_{j=1}^{k} p_{i j}=1 \vee i, j \in\{1, \ldots, k\}, p_{i j} \geq 0 \text { para } i, j \\
& =1,2, \ldots, K
\end{aligned}
$$

Aqui, $p_{i j}$ representa a probabilidade de que, em $t+1$, a cadeia mude do regime $i$ para o regime $j$. A ideia, portanto, é que a probabilidade de ocorrência de um regime $s_{t}$ qualquer, no presente, depende apenas do regime que ocorreu no período anterior, isto é, de $s_{t-1}$. Com $k$ regimes existentes, as probabilidades de transição entre estados podem assim ser representadas pela matriz de transição de probabilidade $\mathrm{P}$, com dimensão $(k x k)$.

Note ainda que a variável TAXA permanece naturalmente endógena, só que desta vez a equação da forma reduzida para essa variável deve ser adaptada para uma versão com mudança de regime tal como se mostra a seguir.

$$
T A X A_{t}=\delta_{0}(s)+\delta_{1}(s) \underset{+}{\operatorname{SELIC}} C_{t}+\delta_{2}(s) \underset{+}{\operatorname{INAD} 90_{t}+v_{t}}
$$

onde $v_{t} \sim N\left(0, \phi^{2}\left(s_{t}\right)\right)$

Para estimar o modelo MS com variável endógena usamos o método proposto por Kim (2004). Pela complexidade e por economia não 
replicaremos a metodologia. Os interessados encontrarão no referido artigo uma descrição sucinta.

Os parâmetros do modelo acima são estimados a partir da maximização da função de verossimilhança do modelo por meio do algoritmo EM (Dempster, Laird e Rubin, 1977), uma técnica iterativa para modelos com variáveis omitidas e/ou não observadas. Pode ser mostrado que o valor da função verossimilhança relevante aumenta a cada iteração desse processo, o que garante que o resultado final seja suficientemente próximo do valor máximo da verossimilhança na vizinhança relevante ${ }^{30}$. É necessário ter em mente, entretanto, que a função de verossimilhança de um modelo MS não possui máximo global (Hamilton, 1991, 1993). Felizmente, a utilização do algoritmo EM frequentemente leva a obtenção de um máximo local "razoável", com casos patológicos sendo relativamente raros (Hamilton, 1993).

\section{Resultados econométricos}

\subsection{Cronologia dos regimes}

Nesta seção apresentamos os resultados do modelo MS, seguindo o procedimento descrito na seção anterior. Quanto ao número de regimes deve ser entendido que o modelo MS é altamente parametrizado, pois permite observar o comportamento dos distintos critérios de escolha admitindo mudanças no intercepto (I), nos parâmetros das variáveis (A) e nas variâncias $(\mathrm{H})$ em cada regime. Tendo em vista o tamanho da amostra, optamos pela estimação do modelo com três regimes (MS(3)-IAH). A introdução de um número superior de regimes conduziu a problemas no processo de otimização ${ }^{31}$ fazendo com que a matriz de transição de probabilidade se tornasse não ergódica, violando uma das principais hipóteses do modelo. Tendo em vista, ainda, a dimensão temporal da série, parece pouco plausível que tenha ocorrido um número de regimes maior que três durante este período. Aplicamos o teste de razão de verossimilhança

(30) Em geral esse método se mostra robusto quando os valores iniciais são estabelecidos de maneira arbitrária ou pouco eficiente.

(31) A série é composta de 149 observações sendo o número de parâmetros 22. 
(LR) ${ }^{32}$ que rejeitou a hipótese nula de linearidade, isto é, um modelo sem mudança de regime ${ }^{33}$. A Figura 1 apresenta os gráficos da cronologia dos regimes (notadamente da trajetória da probabilidade suavizada) ${ }^{34}$ para o modelo MS(3)IAH.

Figura 1

Probabilidades Suavizadas - MS(3)IAH
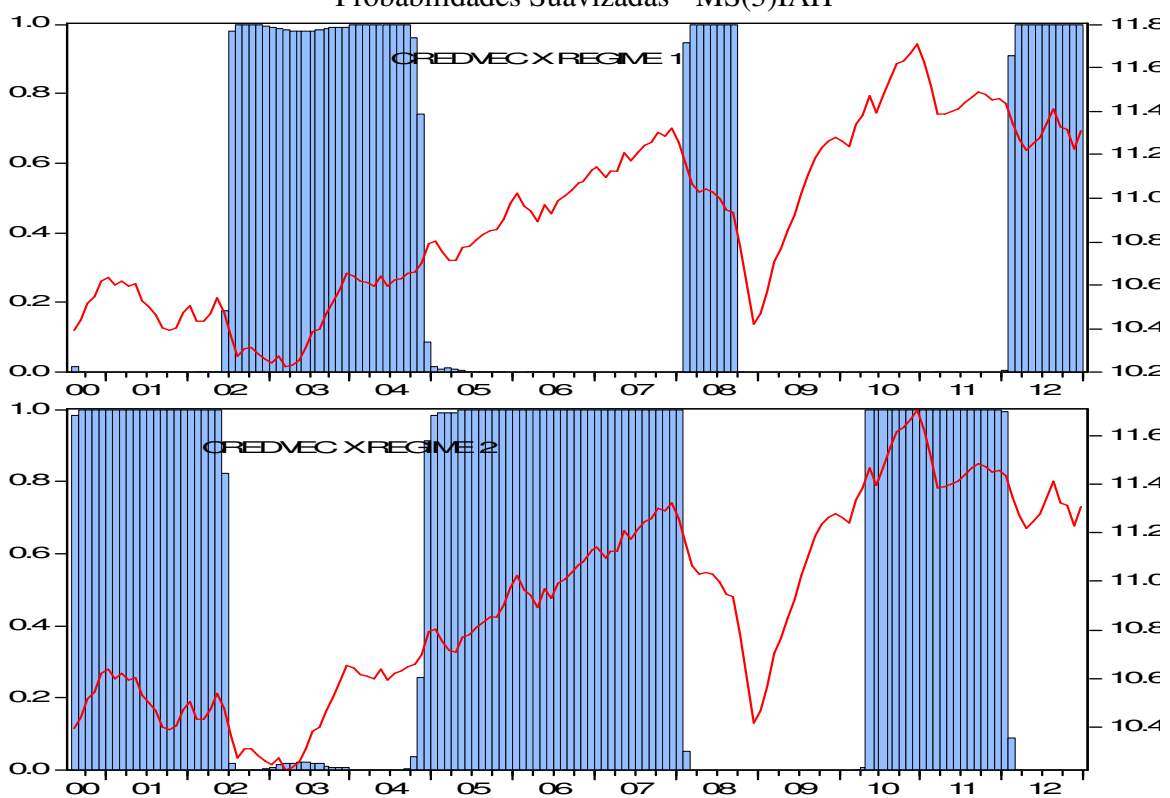

(32) O teste LR aqui possui distribuição não padrão, não podendo ser caracterizado analiticamente desde que as probabilidades de transição são não identificadas sob a hipótese de linearidade. Contudo, é possível mostrar que esta distribuição pode ser aproximada, estando no intervalo entre duas qui-quadrado. Deduz-se disso que, se essas distribuições rejeitarem a hipótese nula, o teste LR deverá necessariamente fazê-lo. Contrariamente, se não houver rejeição da hipótese de linearidade por ambas, então o mesmo deverá acontecer para o teste LR. Em qualquer outra situação, nada poderá ser dito (Davies, 1977; Hansen, 1992).

(33) Teste LR: Chi^2(16) $=289.87[0.0000]^{* *}$

(34) A probabilidade suavizada (smoothed) considera informações de toda a amostra, sendo definida da seguinte forma: $\operatorname{Pr}\left[S_{t}=j \mid \Psi_{T}\right]$, onde $\Psi_{T}$ é o conjunto de informação pleno até o instante T. A probabilidade filtrada (filtered) é uma inferência ótima no estado da variável no tempo $t$, considerando as informações até $t$, enquanto a probabilidade predita (predicted) considera a informação até $t-1$. 


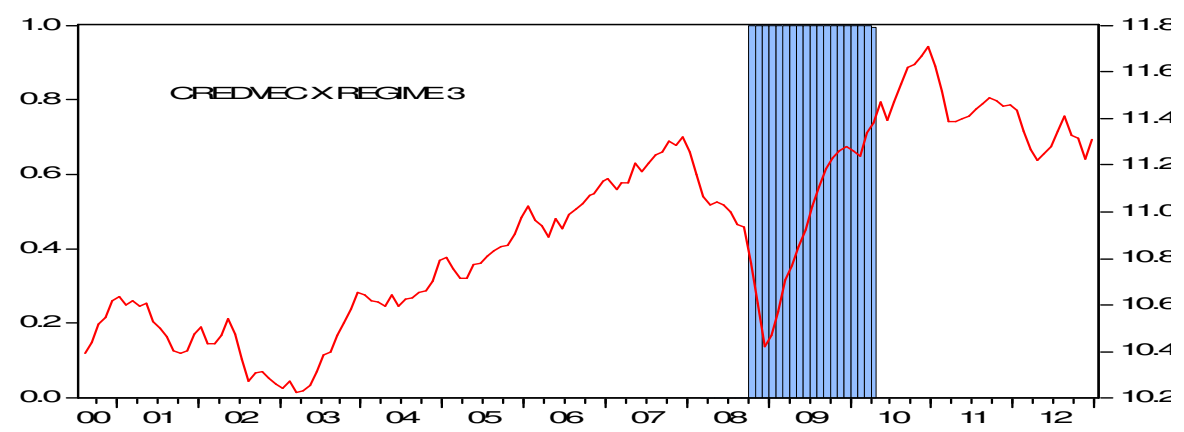

Fonte: Elaboração dos autores

A Figura 1 agrupa os gráficos dos três regimes onde a série de concessão de crédito aparece em cada um dos gráficos. O modelo MS(3)IAH parece captar alguns dos fatos estilizados descritos na seção 2. Note-se a forte coincidência entre as quebras assinaladas heuristicamente no Gráfico 1 que aparece na sessão introdutória deste estudo e as mudanças estruturais apontadas pelo modelo MS.

A Tabela 2 apresenta a cronologia dos regimes. Naturalmente usaremos de certo grau de arbitrariedade de modo a procurar conjugar a datação dos regimes com o estado da economia. Ao regime 1 associamos a fase de retração da atividade creditícia enquanto o regime 2 acreditamos que o regime 2 se relaciona com a fase de expansão do crédito. Pode-se postular que o segundo ciclo do regime 2 guarda relação com as mudanças institucionais na legislação do crédito ocorridas neste período, assim como o bom desempenho da economia neste período contribuiu para a tendência estável de crescimento do crédito durante este regime. Esta fase marca o período de longínqua e robusta expansão do crédito para veículos, que se finda quando entra em cena o segundo ciclo (fase) do regime 2. Este coincide com o surgimento da crise econômica de 2008, impactando diretamente na retração abrupta do crédito. 
Tabela 2

Cronologia dos Regimes

\begin{tabular}{c|c|c|c}
\hline & $1^{\circ} \mathrm{FASE}$ & $2^{\circ} \mathrm{FASE}$ & $3^{\circ} \mathrm{FASE}$ \\
\hline REGIME 1 & $2002(1 \mathrm{~T})-2004(2 \mathrm{~T})$ & $2007(3 \mathrm{~T})-2008(1 \mathrm{~T})$ & $2011(3 \mathrm{~T})-2012(4)$ \\
\hline REGIME 2 & $2000(3 \mathrm{~T})-2001(4 \mathrm{~T})$ & $2004(3 \mathrm{~T})-2007(2 \mathrm{~T})$ & $2009(4 \mathrm{~T})-2011(2 \mathrm{~T})$ \\
\hline REGIME 3 & $2008(2 \mathrm{~T})-2009(3 \mathrm{~T})$ & & \\
\hline
\end{tabular}

Fonte: Elaboração dos autores.

A partir de meados de 2008 tem início o regime 3 e que marca uma nova etapa de expansão do crédito para veículos. Diferente da fase de expansão do credito vista no regime 2 , esta ascensão é bem mais inclinada, sugerindo que a resposta do crédito é bem mais sensível aos incentivos. Vale frisar que, diferente dos demais estados, o regime 3 se compõe de somente um ciclo. Com base na análise elaborada na seção dois, pode-se ver que este período está associado a uma série de medidas editadas pelo governo, com o fim de reaquecer a economia. Entre essas medidas, encontram-se aquelas que tinham como objetivo aumentar a oferta de crédito, como a diminuição dos recolhimentos do compulsório. A queda na taxa Selic também contribui para a retomada do crédito. Foram, ainda, facilitadas as condições para liberação de crédito. Além disso, o governo procurou incentivar o mercado automobilístico diminuindo a alíquota do IPI que vigorou entre dezembro de 2008 a fevereiro de 2010, tal como foi dito na seção 2 .

Foi ainda durante o período em que vigora o regime 3 que o $\mathrm{BCB}$ começou, já no início de 2010, a perceber que o crédito estava se expandindo de uma forma muito rápida. Assim em fevereiro desse ano, numa tentativa de prevenir os riscos desta expansão, o BCB começou a impor medidas macroprudenciais. Inicialmente, isso se deu a partir da recomposição do montante de recolhimentos compulsórios para o nível pré-crise. O fato, porém, é que o crédito continuou se expandindo, o que fez com que o BCB ampliasse as medidas macropudenciais em dezembro de 2010, no que tange a elevação dos compulsórios, diminuição do prazo de financiamento etc., tal como analisado na seção 2 .

Não existe uma proposta cabal acerca da interpretação econômica dos diferentes regimes. Contudo, tendo em vista que a datação deles parece 
estar atrelada a eventos importantes da economia, como a crise mundial de 2008, ou de medidas de política econômica de diversas categorias, pode-se tentar dar um contorno mais preciso ao significado de cada um deles. Começando pelo regime 3, que, como já frisado, se compõe de apenas um ciclo. Para efeito deste estudo, a proposta é que este é um regime de formação de bolha. A justificativa para isso se baseia em que, logo após a eclosão da crise econômica de 2008, o BCB utilizou uma série de medidas anticíclicas com intuito de mitigar o efeito da retração no crédito. Assim, certas medidas foram tomadas com o objetivo de promover a expansão da oferta de crédito, como no caso da diminuição do recolhimento dos compulsórios; e outras atuaram no lado da demanda, como a expansão do prazo de financiamento.

Não entrando no mérito de ser a favor ou contrário ao emprego de medidas anticíclicas, deve-se considerar que, sem que se conheça como interagem as funções de demanda e oferta de crédito, é muito arriscado o emprego de qualquer medida. Portanto, a retração pode ter sido, pelo menos parcialmente, causada por uma queda na demanda por concessões de crédito.

\subsection{Análise das regressões}

Vejamos agora se os resultados obtidos para os coeficientes estimados das regressões para cada estado corroboram as colocações feitas anteriormente com relação não apenas com os sinais esperados assim como com a cronologia dos regimes analisadas na seção anterior. Conforme pode ser visto na Tabela 3, os sinais dos regressores estão em conformidade com o esperado. Um aumento da taxa de financiamento tem efeito negativo sobre as concessões de crédito para todos os regimes. A análise dos resultados obtidos para as variáveis $P R E V C$ assinala aspectos bastante interessantes. Enquanto o sinal obtido para essa variável se mostra em conformidade ao esperado, é visto que no regime 3 o efeito dessa variável medido pelo seu coeficiente é muito superior que nos demais estados. A explicação aqui é simples na medida em que o regime 3 é aquele onde houve forte redução do IPI o que refletiu naturalmente na queda acentuada no preço. Assim, a 
redução do preço do veículo não se deveu nesse regime a um movimento inerente ao mercado, mas sim de fatores exógenos.

Também pode ser percebido que a variável $T X P I B$ não se mostra significativa nesse regime, o que está em conformidade com nossa análise tendo em vista que houve no regime 3 forte intervenção do governo de diversas formas, tal que o estado da atividade econômica deixa de ter efeito sobre a demanda por financiamento. No que se refere às demais variáveis não há muito que se comentar exceto o fato de que seus sinais estão em conformidade com o esperado. Outro ponto que vale menção é o grau de volatilidade ilustrado pelo desvio padrão do erro amostral é menor no regime 3 enquanto se mantém o mesmo nos demais regimes.

Por fim, passando agora à análise da matriz de transição de probabilidades (Tabela 4), nota-se que, uma vez dentro de cada um dos três regimes, existe uma alta probabilidade de permanência. No entanto, o regime 3 apresenta menor probabilidade de ocorrer dado que a economia se mostra distinta em relação aos demais regimes. Isso pode refletir o fato de que, tomando por base que esse regime é autônomo, ou melhor, diferenciado dos demais quanto ao menor grau de liberdade com as forças do mercado atuam, é natural ele seja menos visitado.

Tabela 3

Regressões do MS(3)AIH

Variável dependente: LCONCVC*

\begin{tabular}{l|c|c|c}
\hline & Regime 1 & Regime 2 & Regime 3 \\
\hline CONST & $-6.071(0.000)$ & $0.647(0.845)$ & $28.895(0.000)$ \\
\hline TAXA & $-0.0 .013(0.000)$ & $-0.024(0.000)$ & $-0.099(0.000)$ \\
\hline LPREVCN & $0.901(0.000)$ & $0.122(0.000)$ & $5.431(0.000)$ \\
\hline TXPIB & $0.086(0.000)$ & $0.030(0.000)$ & $-2.937(0.177)$ \\
\hline PRAZO & $-0.001(0.484)$ & $0.002(0.000)$ & $0.005(0.000)$ \\
\hline Desvio-Padrão & $0.054(0.000)$ & $0.053(0.000)$ & $0.046(0.000)$ \\
\hline
\end{tabular}

Nota: P-valor entre parênteses. L: transformação logarítmica.

Fonte: Elaboração dos autores. 
Tabela 4

Probabilidades de Transição

\begin{tabular}{l|c|c|c}
\hline & \multicolumn{3}{|c}{ Em t+1 } \\
\hline \multicolumn{1}{c|}{ Em t } & Regime 1 & Regime 2 & Regime 3 \\
\hline Regime 1 & 0.955 & 0.023 & 0.021 \\
\hline Regime 2 & 0.036 & 0.963 & 0.000 \\
\hline Regime 3 & 0.000 & 0.055 & 0.945 \\
\hline
\end{tabular}

Fonte: Elaboração dos autores.

\section{Comentários finais}

Este estudo teve como objetivo estimar uma função de demanda por crédito para veículos no Brasil. Tomamos por hipótese que a série de concessão de crédito para veículos representa um conjunto de pontos formados pela interação entre a demanda e oferta de financiamento de veículos onde a "quantidade" (concessão de crédito) e o preço (taxa de financiamento) são determinados endogenamente. O problema da endogeneidade foi analisado tomando por base os métodos tradicionais (2SLS/IV/GMM) que apontaram certa endogeneidade no modelo.

Nossos resultados mostraram que foi possível identificar a função de demanda por financiamento na qual o preço do veículo, a taxa e prazo de financiamento assim como o estado da economia são as variáveis explicativas do modelo, ou seja, a função de demanda por concessão de crédito para veículo segue de perto o que se espera de uma função de demanda tradicional.

Mostramos ainda com base no modelo Markov Switching (MS) com variável endógena (Kim, 2004) que tal função de demanda esteve sujeita a três regimes distintos ao longo do período amostral. Dois deles estão associados aos regimes de expansão e retração que são inerentes a uma economia de mercado no qual o estado da economia é determinado pelos fundamentos. Por exemplo, um ciclo de forte expansão acontece entre 2004 e 2007 enquanto um ciclo de retração vigora entre o final de 2007 e início de 2008.

Destaca-se ainda a existência de um terceiro regime também de expansão, ocorrido num único ciclo entre 2008 e 2009 e que foi, no nosso 
entendimento, gerado de modo artificial em decorrência do emprego de medidas anticíclicas fomentadas pelo governo. Entre elas, pode-se destacar a redução da base de recolhimento do compulsório e mudança da alíquota do IPI assim como o aumento do prazo de financiamento. Essas medidas levaram à imediata expansão do crédito e a um processo de formação de bolha no mercado de veículos, que culminou com a necessidade de introdução das chamadas medidas macroprudenciais, a partir de dezembro de 2010, quando já havia em curso uma tendência de contínuo aumento da inadimplência. Sendo assim, o emprego de uma política anticíclica deve considerar suas consequências adversas antes de ser visto como um meio de mitigar os efeitos da fase recessiva do ciclo econômico.

\section{Referências bibliográficas}

ALVARENGA, ALVES, P.; SANTOS, C.; NEGRI, F.; CAVALCANTI, L.; PASSOS, M. Políticas anticíclicas na indústria automobilística: uma análise de cointegração dos impactos da redução do IPI sobre as vendas de veículos. Rio de Janeiro: Ipea, 2010. (Texto para Discussão, n. 1.512).

ARAÚJO, V. L. Preferência pela liquidez dos bancos públicos no ciclo de expansão do crédito no Brasil: 2003-2010. Brasília: Ipea, 2012. (Texto para Discussão, n. 1.717).

ASSUNÇÃO, J.; BENMELECH, E., SILVA, F. Repossession and the democratization of credit. Massachusetts: National Bureau of Economic Research, 2012. (NBER Working Paper, n. 17.858).

BLUM, D.; NAKANE. M. I. O impacto de requerimentos de capital na oferta de crédito bancário no Brasil. In: BCB - BANCO CENTRAL DO BRASIL. Relatório de economia bancária e crédito. Brasília: BCB, 2006.

BOUND, J.; JAEGER, D. A.; BAKER, R. Problems with instrumental variables estimation when the correlation between instruments and endogenous variables is weak. Journal of the America Statistical Association, v. 90, p. 443-450, 1995.

CHIB, S. Calculating posterior distributions and modal estimates in Markov mixtures models. Journal of Econometrics, v. 75, p. 79-97, 1996. 
COOK, S. Spurious rejection by cointegration tests incorporating structural change in the cointegrating relationship. Applied Economics Letters, v. 11, 879-884, 2004.

DAVIES, R. B. Hypothesis testing when a nuisance parameter is present only under the alternative. Biometrika, v. 64, n. 2, p. 247-254, 1977.

DAWID, P. E. Liberação de compulsórios, crédito bancário e estabilidade financeira no Brasil. Brasília: BCB, 2009. (Relatório de Economia Bancária e Crédito).

DAWID, P. E.; TAKEDA, T. Recolhimentos compulsórios e o crédito bancário no Brasil. Brasília: Banco Central do Brasil, 2012. (Texto para Discussão, n. 250).

DEMPSTER, A. P.; LAIRD, N. M.; RUBIN, D. B. Maximum likelihood from incomplete data via the EM algorithm. Journal of Royal Statistical Society, v. 39, p. 1-38, 1977.

FRANCES, P. H.; DIJK, D. V. Non-linear time series models in empirical finance. Cambridge: Cambridge University Press, 2000.

HANSEN, B. E. The likelihood ratio test under nonstandard assumptions: testing the Markov switching Model of GDP. Journal of Applied Econometrics, v. 7, p. S61-S82, 1992. (Erratum, v. 11, p. 195-198, 1996).

HAMILTON, J. A Quasi-Bayesian approach to estimating parameters for mixtures of normal distributions. Journal of Business and Economic Statistics, v. 9, n. 1, p. 27-39, 1991.

HAMILTON, J. Time series analysis. Princeton: Princeton University Press, 1993.

KIM, C. Markov switching model with endogenous explanatory variables. Journal of Applied Econometrics, v. 122, p. 127-136, 2004.

KIM, C.; NELSON, C. State-space models with regime switching: classical and Gibbs-sampling approaches witch applications. Cambridge, Mass.: MIT Press, 1999. 
KROLZIG, H. M. Markov-Switching vector autoregressions. Modelling, statistical inference and application to business cycle analysis. Berlin: Springer-Verlag, 1997.

LEYBOURNE, S.; NEWBOLD, P. Spurious rejections by cointegration tests induced by structural breaks. Applied Economics, v. 35, p. 1117-1121, 2003.

LUTKEPOHL, H.; JUDGE, G. G.; HILL, R. C.; GRIFFITHS, W. E. ; LEE, T.-C. Theory and practice of econometrics, $2^{\text {nd }}$. ed. New York: John Wiley \& Sons, 1985.

MARTINS, B. Crise financeira e restrição de crédito no Brasil. Brasília: $\mathrm{BCB}, 2010$. (Relatório de Economia Bancária e Crédito).

MARTINS, B. S.; LUNDBERG, E. L.; TAKEDA, T. Crédito habitacional no Brasil: aperfeiçoamentos institucionais e avaliação da evolução recente. Brasília: BCB, 2008. (Relatório de Economia Bancária e Crédito).

MENDONÇA, M. J. C.; SACHSIDA, A. Identificando a demanda e oferta de crédito no Brasil. Rio de Janeiro: Ipea, 2013. (Texto para Discussão, n. 1.837).

MOREIRA, Marcelo J. Conditional likelihood ratio test for structural models. Econometrica, v. 71, n. 4, p. 1027-1048, 2003.

MURRAY, M. P. Avoiding invalid instruments and coping with weak instruments. Journal of Economic Perspectives, v. 20, p. 111-132, 2006.

$\mathrm{NOH}$, J.; KIM, T. Behaviour of cointegration tests in the presence of structural breaks in variance. Applied Economics Letters, v. 10, p. 9991002, 2003.

PESARAN, M. H.; TAYLOR, L. W. Diagnostic for IV regressions. Oxford Bulletin of Economics and Statistics, v. 61, n. 2, p. 255-281, 1999.

SHEA, J. Instrument relevance in multivariate linear models: a simple measure. Review of Economics \& Statistics, v. 79, p. 348-352, 1997.

SIMS, C. Stability and instability in US monetary policy behavior. Princeton: Princeton University, 2001. (Discussion Paper). 
SOUZA, M. N. Carneiro de. Credit restrictions and occupational choice: the impact of Payroll Lending. Essays on banking. 2011. Tese (Doutorado)Pontifícia Universidade Católica do Rio de Janeiro, Rio de Janeiro, 2011. STOCK, J. H; YOGO, M. Testing for weak instruments in linear IV regression. In: ANDREWS, D. W.,K.; STOCK, J. H. (Ed.). Identification and inference for econometric models. Essays in Honor of Thomas Rothenberg. New York: Cambridge University Press, 2005. p. 80-108.

WOOLDRIDGE, J. Econometric analysis of cross section and panel data. Cambridge, Mass.: The MIT Press, 2002. 